DOE/NV/11718--1050

\title{
SUITABILITY OF MAGNESIUM OXIDE AS A VISAR WINDOW
}

\author{
G. D. Stevens ${ }^{1}$, L. R. Veeser ${ }^{1}$, P. A. Rigg ${ }^{2}$, R. S. Hixson ${ }^{2}$ \\ ${ }^{1}$ Bechtel Nevada, Special Technologies Laboratory, Santa Barbara CA 93111 \\ ${ }^{2}$ Los Alamos National Laboratory, Los Alamos, NM 87545
}

\begin{abstract}
Impedance matching of a velocity interferometer for any reflector (VISAR) window to a material under study helps simplify a shock experiment by effectively allowing one to measure an in situ particle velocity. The shock impedance of magnesium oxide $(\mathrm{MgO})$ falls roughly midway between those of sapphire and $\mathrm{LiF}$, two of the most frequently used VISAR window materials. A series of symmetric impact experiments was performed to characterize the suitability of single crystal, (100) oriented magnesium oxide as a VISAR window material. These experiments yielded good results and show the viability of $\mathrm{MgO}$ as a VISAR window up to $23 \mathrm{GPa}$. Results were used to determine window correction factors and, subsequently, to estimate the pressure induced change in index of refraction. In many of the shots in this work we exceeded the Hugoniot elastic limit (HEL) of $\mathrm{MgO}$, and both elastic and plastic waves are evident in the velocity profiles. The presence of both waves within the VISAR window complicates the typical VISAR window correction analysis. Preliminary analysis of the elastic and plastic contributions to the window correction is presented.
\end{abstract}

Keywords: Interferometer, magnesium oxide, shock wave, VISAR

PACS: $47.40 . \mathrm{Nm}, 62.50 .+\mathrm{p}, 78.20 . \mathrm{Ci}, 42.62 . \mathrm{Eh}$

\section{INTRODUCTION}

We have performed a study of shock effects on single-crystal $\mathrm{MgO}$ to characterize it for possible use as a window in shock-wave experiments. A transparent window on the back of a shocked sample can allow measurements of its properties, such as wave profiles, with greatly-reduced shock reflection and unloading effects if the window impedance is closely matched to that of the sample. The shock wave impedance (density times sound speed) of $\mathrm{MgO}$ is roughly midway between those of sapphire and $\mathrm{LiF}$, two of the most important window materials. Consequently, if its other properties are suitable, $\mathrm{MgO}$ also could be a very useful window because its shock impedance moreclosely matches some sample materials.

To make a good interferometer window, a material must remain transparent up to at least the shock pressures of interest in the sample being studied. The window material should not have phase-change-induced problems over the pressure range of interest, it should not fracture, and it should be chemically and physically stable and easy to handle. In addition, it is important to understand how its refractive index behaves when the crystal is compressed by a shock because if the refractive index changes, the wavelength of the light in the crystal also changes, thereby affecting the VISAR signal.

In this experiment we used a VISAR [1,2] to measure material velocities. In a VISAR experiment laser light is reflected from a moving surface. A window between the sample and the light collection system changes the properties of the Doppler-shifted, reflected light [3]. A shock in the window can change its refractive index, complicating the data interpretation. This change, 
which must be accounted for and corrected, is described either as a change in the apparent velocity, $\Delta \mathrm{u}=\mathrm{u}_{\mathrm{a}}-\mathrm{u}_{0}$, or a multiplicative factor, $\mathrm{u}_{\mathrm{a}} / \mathrm{u}_{0}=1+\Delta \mathrm{v} / \mathrm{v}_{0}$.

Here $u_{a}$ is the apparent velocity of the samplewindow interface measured by the VISAR; $\mathrm{u}_{0}$ is its actual velocity, the particle velocity at the interface; $v_{0}$ is the Doppler-shifted frequency of light scattered from the moving interface; and $\Delta v$ represents the change in frequency of the reflected light because of the shock in the window.

In this paper we report measurements of apparent window velocities from shock waves in $\mathrm{MgO}$ windows. For most of our measurements a VISAR measured the velocity of the interface between a $\mathrm{MgO}$ sample and a $\mathrm{MgO}$ window following impact of a $\mathrm{MgO}$ flyer onto the sample. The technique is similar to that of Jones et. al [4]. For a symmetric-impact experiment like this, the particle velocity of the shock wave in the sample is exactly half that of the flyer at impact. The flyer velocity and tilt are measured with a set of about 12 electrical shorting pins around the sample. The ratio of the interface velocity measured with the VISAR to the particle velocity deduced from the pins is the window correction factor, $\mathrm{u}_{\mathrm{a}} / \mathrm{u}_{0}$, described in the preceding paragraph.

\section{EXPERIMENTAL PROCEDURE}

Fig. 1 shows a schematic of the experimental setup. Five symmetric-impact $\mathrm{MgO}$ gun experiments, where the window was also of $\mathrm{MgO}$, were performed. The two lower-pressure measurements were made at the Los Alamos National Laboratory TA-39 Popgun, and the three higher-pressure measurements were made at the powder gun at TA-40. Having a wide range of gun velocities available allowed us to avoid using higherimpedance flyers or samples to reach the highest pressures. By using $\mathrm{MgO}$ for flyer, sample, and window, we avoid mixing effects in $\mathrm{MgO}$ with those of other materials and thus make the experiment easier to analyze and understand. The $\mathrm{MgO}$ flyers, samples, and windows were single crystal material oriented along the [100] direction.
The samples were obtained from MTI Corporation, and were $99.95 \%$ pure with average densities of $3.58 \mathrm{~g} / \mathrm{cm}^{2}$. The interface between the sample and the window had a thin reflective aluminum layer to reflect light from a frequency-doubled YAG laser (532 nm wavelength) for velocity measurement in a pair of VISARs. The VISARs measured the

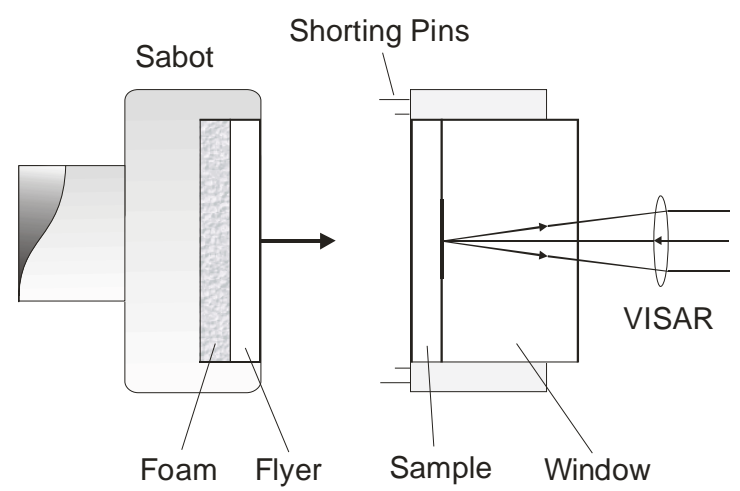
apparent interface velocity to between 1 and $2 \%$.

Figure 1. Experimental arrangement in which the flyer was accelerated by a gun. The flyer, sample, and window were all of $\mathrm{MgO}$. The center of the interface between the sample and window has a thin coating of aluminum to allow reflection of laser light into a VISAR. A ring of 11 shorting pins and one piezoelectric trigger pin around the sample surface give a measurement of the flyer velocity and tilt at impact.

In addition to the gun shots, we performed two explosively-driven experiments. Each consisted of a 12-mm-thick, 12.7-mm-diameter Detasheet highexplosive (HE) driver and a 2-mm-thick, 20-mmdiameter copper sample backed by a 5 -mm-thick, 20-mm-diameter (100)-oriented $\mathrm{MgO}$ window. Measurements were made of the copper-window interface and, in separate experiments, of the same driver and copper sample without a window and with a $\mathrm{LiF}$ window. This arrangement is not planar, the shock wave is not flat-topped, and the sample is not of $\mathrm{MgO}$, but we believe that the results are relevant and we include them here for completeness. Table 1 summarizes the nominal geometrical dimensions, velocities, and stresses of all the measurements. 
DOE/NV/11718--1050

TABLE 1. Experimental configurations

\begin{tabular}{lcccccc}
\hline $\begin{array}{c}\text { Experiment, } \\
\text { Facility }\end{array}$ & $\begin{array}{c}\mathbf{u}_{\text {sabot }} \\
(\mathbf{k m} / \mathbf{s})\end{array}$ & $\begin{array}{c}\text { Stress } \\
(\mathbf{G P a})\end{array}$ & $\begin{array}{c}\text { Diameter } \\
(\mathbf{m m})\end{array}$ & $\begin{array}{c}\text { Flyer } \\
(\mathbf{m m})\end{array}$ & $\begin{array}{c}\text { Sample } \\
(\mathbf{m m})\end{array}$ & $\begin{array}{c}\text { Window } \\
(\mathbf{m m})\end{array}$ \\
\hline a) powder gun & 1.6474 & 22.9 & 32 & 3 & 3 & 12 \\
b ) powder gun & 1.4537 & 19.9 & 32 & 3 & 3 & 12 \\
c) powder gun & 1.2360 & 16.6 & 32 & 3 & 3 & 12 \\
d) gas gun & $\sim 0.6($ est.) & $\sim 7.6$ & 38 & 3 & 3 & 4.5 \\
e) gas gun & 0.2193 & 2.7 & 38 & 3 & 3 & 4.5 \\
\hline
\end{tabular}

\section{RESULTS}

The results from the gun experiments are shown in Fig. 2. Only one of the two VISAR measurements is presented for each curve, since agreement between the two measured curves is very good. Curves a), b), and c) are for the powder gun experiments. Each exhibited a small but welldefined elastic precursor followed by a jump to a maximum apparent velocity. When the rarefaction from the back of the flyer arrives at the samplewindow interface, it slows the interface. At these high stresses the reflected precursor and the reflected main shock are partially merged by this time, so the rarefaction curves have lost most of the distinction between the precursor and the main shock, and the velocity decreases slowly at first. The final velocity is somewhat above zero because the foam backing on the flyer partially reflects the shock. Again, the maximum apparent velocity is larger than the particle velocity because of the shock in the window.

Results for the two low-velocity gas gun shots are also shown in Fig. 2 (traces $d$ and e). The curves are the apparent velocities measured by the VISARs. Experiment d) was slightly above the elastic limit and e) was below. Both had relatively thin, 4.5-mm windows to allow the elastic shocks to pass completely through them before the rarefactions from the foam backing behind the flyer reached the sample-window interfaces viewed by the VISARs. For experiments d) and e), the elastic shocks unload at around $1.7 \mu \mathrm{s}$ and $1.9 \mu \mathrm{s}$ respectively. The changes in VISAR signals at these times are not caused by an actual interfacevelocity change but by a window-correction change. When the elastic shock unloads, its reflection reduces the stress and increases the particle velocity in the window. This change causes the window correction, $\Delta \mathrm{u}=\mathrm{u}_{\mathrm{a}}-\mathrm{u}_{0}$, to decrease,[4] and we see an apparent velocity drop. The decrease in $u_{a}$ is nearly as large as the window correction at early times, and the resulting window correction is approximately the negative of what it was previously. Knowing the magnitude of the window-correction change gives a useful check on the measurement of its value and confirms that the window correction for this shot is not the same as the correction at pressures above the HEL. Notice also that for experiment e) in Fig. 2, the velocity after shock release appears to become negative at some point; this, too, is caused by the window correction, which at this time has $\Delta \mathrm{u}<0$.

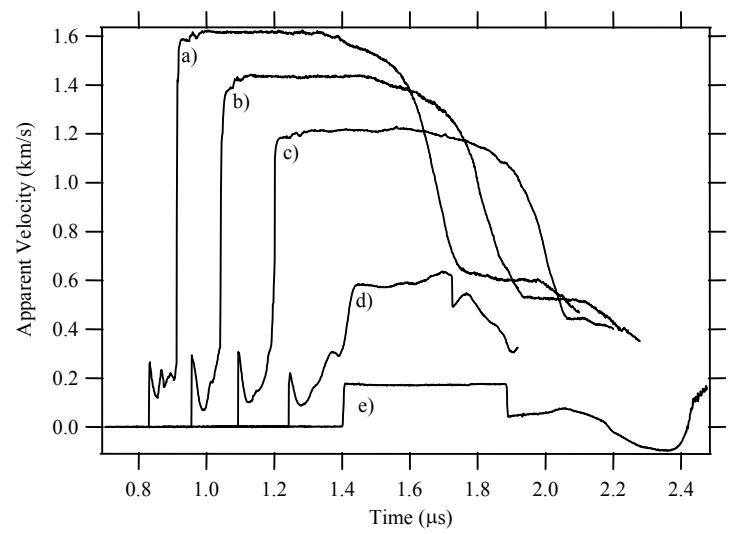

Figure 2. Apparent velocities of the sample-window interface for all five gun experiments. Impact velocities and relevant shot parameters are in Tables 1 and 2 . The apparent velocities shown have not been corrected for the effects of the shock in the window. Relative timing of the five traces has been adjusted for figure clarity.

The results for two separate explosive-driven experiments are shown in Fig. 3, along with a third, similar shot using a $\mathrm{LiF}$ window instead of the $\mathrm{MgO}$. The $\mathrm{MgO}$ apparent-velocity data are divided by 1.978 , which is approximately its window correction. To make the two curves easy to 
compare, the particle-velocity data for the $\mathrm{LiF}$ window are divided by 1.18 , which is the ratio of the interface velocities for the two window types as calculated from their equations-of-state. From this representation, it appears that that the release is much smoother for the LiF anvil than the release into $\mathrm{MgO}$. Effects of this type may call for caution in choosing $\mathrm{MgO}$ windows for some experimental configurations. The sudden drops in velocity at 0.52 and $0.54 \mu$ s are from changes in the window correction when the elastic precursor releases into air. The reason why there are two such pulses is not known at this time.

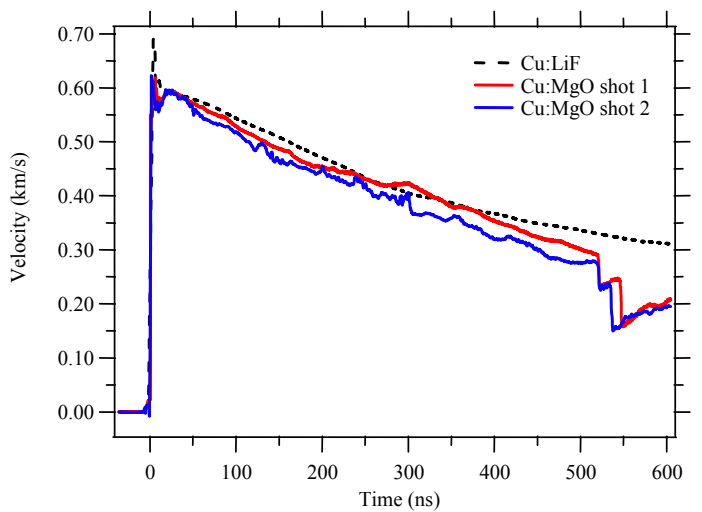

Figure 3. The two solid curves are measurements of a $\mathrm{Cu}-\mathrm{MgO}$ interface velocity with an explosive drive. The top dashed curve is a $\mathrm{Cu}-\mathrm{LiF}$ interface velocity with a similar explosive drive. Curves have been normalized as described in text.

The reproducibility of the shock parameters for copper in this HE-driven system was checked by doing numerous such shots, as well as shots without a window. Without a window, the peak copper velocity at the back surface is $1.00 \mathrm{~km} / \mathrm{s}$. Putting a $\mathrm{LiF}$ window on the back drops the interface velocity to $0.708 \mathrm{~km} / \mathrm{s}$. Replacing the $\mathrm{LiF}$ window with $\mathrm{MgO}$, which has a higher shock impedance, makes the peak interface velocity $0.602 \mathrm{~km} / \mathrm{s}$.

\section{ANALYSIS}

For pressures exceeding the HEL, MgO exhibits clear two-wave structure. In $\mathrm{LiF}$ the HEL is only about $0.2 \mathrm{GPa}$, so experiments are often overdriven and have only a plastic wave structure. For $\mathrm{MgO}$, the HEL is much higher. Duffy and
Ahrens [5] report a value of $1.6 \mathrm{GPa}$; our shot at 2.7 GPA shows no sign of a plastic wave. Because of the high HEL, a strong elastic precursor is present in all of our gun-based, symmetric-impact experiments, which extend up to $23 \mathrm{GPa}$. To model our complete data set adequately, we found it necessary to treat the elastic- and plastic-waveinduced index changes separately, using a different index of refraction relation for each wave. Both indices were assumed to be linear with density.

If $\mathrm{n}_{0}$ is the index of refraction of a window at $532 \mathrm{~nm}$ and $\mathrm{L}$ is the window thickness (Fig. 4), then a two-wave, elastic-plastic shock traveling from the left with velocities $D_{e}$ and $D_{p}$, has an optical thickness, $Z$, which changes with time $t$ as,

$$
Z=n_{0}\left(L-D_{e} t\right)+n_{e}\left(D_{e} t-D_{p} t\right)+n_{p}\left(D_{p} t-u_{0} t\right)
$$

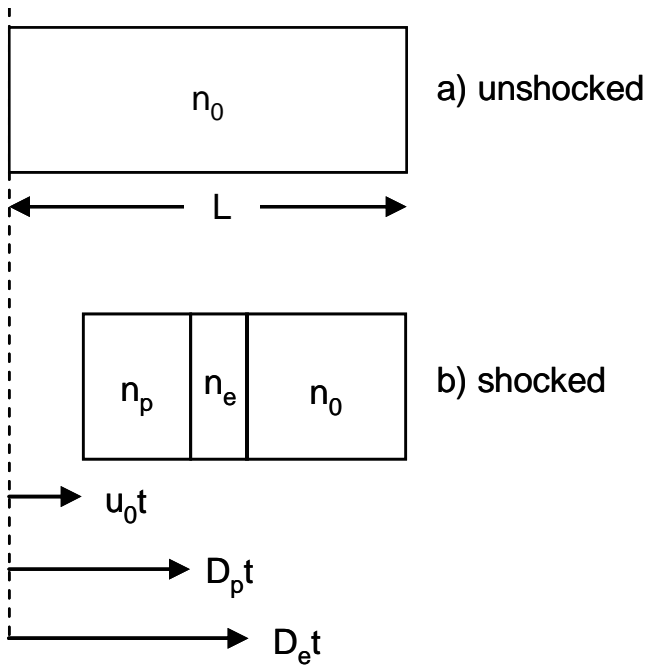

Figure 4. Schematic showing how to calculate the optical thickness of the window. The shock enters from the left. The left edge moves with velocity $\mathrm{u}_{0}$, the particle velocity, while the elastic precursor and the plastic shock travel through the crystal with speeds $D_{e}$ and $\mathrm{D}_{\mathrm{p}}$, respectively. The optical thickness, $\mathrm{Z}$, is given in the text.

The index of refraction of the window has three distinct values in such a situation, $\mathrm{n}_{0}, \mathrm{n}_{\mathrm{e}}$, and $\mathrm{n}_{\mathrm{p}}$. For a symmetric impact experiment where the flyer, sample, and window are of the same material, the particle velocity, $\mathrm{u}_{0}$, behind the main shock is half the impact velocity. The apparent velocity, $u_{a}$, 
of the interface (the left edge of the window) for this two-wave structure is

$$
\mathrm{u}_{\mathrm{a}}=-\mathrm{dZ} / \mathrm{dt}=\left(\mathrm{n}_{0}-\mathrm{n}_{\mathrm{e}}\right) \mathrm{D}_{\mathrm{e}}+\left(\mathrm{n}_{\mathrm{e}}-\mathrm{n}_{\mathrm{p}}\right) \mathrm{D}_{\mathrm{p}}+\mathrm{n}_{\mathrm{p}} \mathrm{u}_{\mathrm{o}}
$$

The actual interface velocity is $u_{0}$, and the difference is:

$$
\begin{aligned}
\Delta u_{p} & =u_{a}-u_{0} \\
& =\left(n_{0}-n_{e}\right) D_{e}+\left(n_{e}-n_{p}\right) D_{p}+\left(n_{p}-1\right) u_{0}
\end{aligned}
$$

For the index of refraction we assume a density dependence of the form $n=A+B \rho$ where $\mathrm{B}=\left(\mathrm{n}_{0}-\mathrm{A}\right) / \rho_{0}$ and $\rho_{0}$ is the density of the unshocked window. We allow for different values of A for elastic and plastic waves. Then the indices are, respectively,

$$
\begin{aligned}
& \mathrm{n}_{\mathrm{e}}=\mathrm{A}_{\mathrm{e}}+\left(\mathrm{n}_{0}-\mathrm{A}_{\mathrm{e}}\right) \rho_{\mathrm{e}} / \rho_{0}, \\
& \mathrm{n}_{\mathrm{p}}=\mathrm{A}_{\mathrm{p}}+\left(\mathrm{n}_{\mathrm{o}}-\mathrm{A}_{\mathrm{p}}\right) \rho_{\mathrm{p}} / \rho_{0} .
\end{aligned}
$$

Neglecting the effects of shear components of stress on the compressed volume, the densities in the two regions behind the elastic and plastic waves are given by

$$
\begin{aligned}
& \rho_{e}=\rho_{0} D_{e} /\left(D_{e}-u_{0 e}\right), \\
& \rho_{p}=\rho_{0} D_{p} /\left(D_{p}-u_{0}\right) .
\end{aligned}
$$

Here $\mathrm{u}_{0 \mathrm{e}}$ is the particle velocity behind the elastic precursor. For a simple elastic wave with no plastic wave, $A_{e}=u_{a} / u_{0 e}$. We only performed one experiment below the HEL, the low velocity popgun shot $\mathrm{e}$ ), which gives $\mathrm{A}_{\mathrm{e}}=0.172 / 0.1096=1.57$.
Using this value we calculate $\mathrm{u}_{0 \mathrm{e}}$ and $\mathrm{n}_{\mathrm{e}}$ for the elastic precursors seen in the higher pressure shots (see Table 2).

We obtained $D_{p}$ for each shot from the Hugoniot equation of state, $D_{p}=6.64+1.35 u_{0}$ [9]. The $\mathrm{D}_{\mathrm{e}}$-values are obtained from the two gas gun experiments, which both gave $D_{e}=9.34 \mathrm{~km} / \mathrm{s}$. Due to scatter in the data, $\mathrm{u}_{\mathrm{ae}}$ was chosen as the average apparent elastic precursor velocity, $\mathrm{u}_{\mathrm{ae}}=0.261$. Using these values and Equations (4) and (5), we determined $A_{p}$ by fitting it to the experimentallydetermined $\Delta \mathrm{u}_{\mathrm{p}}$-values for each shot. This fit gives $\mathrm{A}_{\mathrm{p}}=1.978(1)$. Table 2 lists the results of the fit, and a graph of the fit is shown in Fig. 5.

Hayes [6] reports that the VISAR window correction ratio, $\mathrm{u}_{\mathrm{a}} / \mathrm{u}_{0}$, is constant whenever the index of refraction in the shocked medium, $n(\rho)$, is well described by a linear function of the density, $\rho$. That is, $u_{a} / u_{0}$ is a constant if $n(\rho)=A+B \rho$. Hayes also shows for general $n(\rho)$ that

$$
\mathrm{du}_{\mathrm{a}} / \mathrm{du}_{0}=\mathrm{n}-\rho(\mathrm{dn} / \mathrm{d} \rho)
$$

For a single shock wave propagating in a medium with a refractive index that is linear in density, it follows that $\mathrm{A}=\mathrm{u}_{\mathrm{a}} / \mathrm{u}_{0}$ and $\mathrm{B}=\left(\mathrm{n}_{0}-\right.$ A) $/ \rho_{0}$. Since in this particular case $u_{a} / u_{0}$ is a constant, it follows that $\mathrm{du}_{\mathrm{a}} / \mathrm{du}_{0}=\mathrm{A}$. Using this relation, and evaluating the above expression at a specific wavelength,

$$
A=n-\rho(d n / d \rho)
$$

TABLE 2. Shot parameters and results. $u_{0}$ is one half of the impactor velocity $u_{\text {sabot }} \cdot u_{a e}$ is the apparent elastic precursor velocity, and $u_{a p}$ is the apparent plastic wave velocity. $D_{e}$ and $D_{p}$ are the average elastic wave velocity and the calculated plastic shock wave velocities. $n_{e}$ and $n_{p}$ are the indicies of refraction of the regions behind the elastic and plastic waves respectively. $\Delta \mathrm{u}=\mathrm{u}_{\mathrm{ap}}-\mathrm{u}_{0}$.

\begin{tabular}{cccccccccc}
\hline Expt. & $\mathbf{u}_{\mathbf{0}}$ & $\begin{array}{c}\mathbf{u}_{\mathbf{a e}} \\
\text { (meas.) }\end{array}$ & $\begin{array}{c}\mathbf{D}_{\mathbf{e}} \\
\mathbf{( k m / s )}\end{array}$ & $\mathbf{n}_{\mathbf{e}}$ & $\begin{array}{c}\mathbf{u}_{\mathbf{a p}} \\
\text { (meas.) }\end{array}$ & $\begin{array}{c}\mathbf{D}_{\mathbf{p}} \\
(\mathbf{k m} / \mathbf{s})\end{array}$ & $\mathbf{n}_{\mathbf{p}}$ & $\begin{array}{c}\Delta \mathbf{u} \\
\text { (meas.) }\end{array}$ & $\begin{array}{c}\Delta \mathbf{u} \\
\text { (calculated) }\end{array}$ \\
\hline a) & 0.825 & 0.285 & 9.37 & 1.745 & 1.616 & 7.770 & 1.713 & 0.795 & 0.802 \\
b) & 0.725 & 0.220 & 9.37 & 1.745 & 1.440 & 7.633 & 1.717 & 0.715 & 0.705 \\
c) & 0.620 & 0.328 & 9.37 & 1.745 & 1.215 & 7.489 & 1.720 & 0.600 & 0.600 \\
d) & 0.3 & 0.210 & 9.37 & 1.745 & 0.58 & 7.051 & 1.731 & 0.27 & 0.287 \\
e) & 0.1096 & 0.172 & 9.37 & 1.744 & - & - & - & 0.062 & 0.062 \\
\hline
\end{tabular}




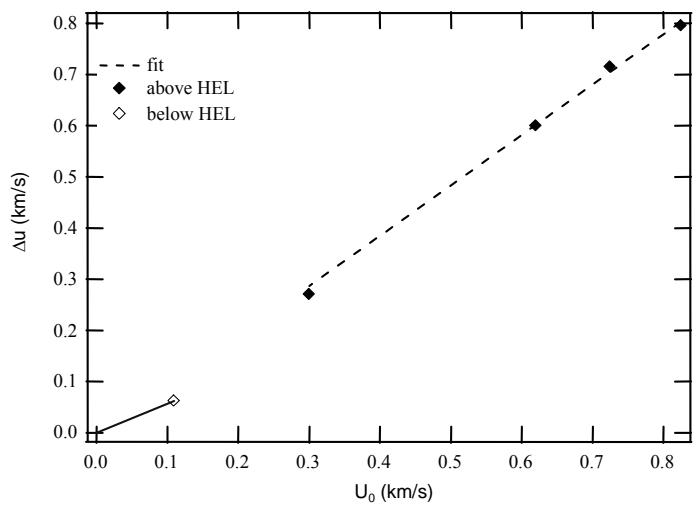

Figure 5. $\mathrm{MgO}$ window-correction measurements. The data above the HEL were fit using $\mathrm{A}_{\mathrm{e}}=1.57$, and $\mathrm{D}_{\mathrm{e}}=$ 9.34, giving $\mathrm{A}_{\mathrm{p}}=1.978(1)$. The point at $\mathrm{u}_{0}=0.3$ (experiment $\mathrm{d}$ ) has a large velocity uncertainty and was weighted accordingly.

It is possible to estimate the window correction for $\mathrm{MgO}$, as well as other materials with an index that is linear in density, from this equation by evaluating it for unshocked material at the wavelength of interest. The refractive index is well known, and the second term is commonly measured for optical materials [7],[8]. Table 3 gives values of this "window correction factor" for a variety of VISAR windows, along with the correction measured by other means, such as that presented in this work. It is interesting to note that the simple method of estimating A gives reasonably good agreement with experimentally determined window correction factors for windows shocked above their HEL. The estimation fails, however, for windows such as sapphire and quartz that are used within their elastic limits.

Vedam [7] has made hydrostatic compression measurements below $0.7 \mathrm{GPa}$, which interpolate to $\rho \mathrm{dn} / \mathrm{d} \rho \approx-0.267$ at $\lambda=532 \mathrm{~nm}$. Consequently for Vedam's data $\mathrm{A}=\mathrm{n}_{0}-\rho \mathrm{dn} / \mathrm{d} \rho=2.007$, close to our value from our measurements above the HEL. Above the HEL, stress is isotropic, and thus the hydrostatic measurements of Vedam may apply in this regime. Below the HEL, stress is uniaxial, and one might expect to find different values of $\rho \mathrm{dn} / \mathrm{d} \rho$ under these conditions.

Our lowest-pressure gun experiment, e), which was the only measurement with a purely elastic shock, gave $\mathrm{A}=1.56$. This lower value of $\mathrm{A}$ is interesting because it implies that $\rho \mathrm{dn} / \mathrm{d} \rho$ is positive for uniaxial, elastic compression, while it is negative for hydrostatic, isotropic compression. Alternately, this may indicate that the index of refraction is not simply linear in density for elastic shocks in $\mathrm{MgO}$.

\section{CONCLUSIONS}

We have measured the window correction for single-crystal $\mathrm{MgO}$ at stresses up to $23 \mathrm{GPa}$. The correction factor appears to be approximately constant with particle velocity except for a single point below the elastic limit. $\mathrm{MgO}$ remains transparent at these pressures, and may be used effectively on gas-gun experiments. The wave profiles are not smooth however, for two dimensional shocks generated by explosives.

Table 3. VISAR window corrections, A, calculated from tabulated values of $\rho(\mathrm{dn} / \mathrm{d} \rho)$ versus values determined by gasgun experiments. Note that the values obtained from hydrostatic measurements contained in references [8], [11], and [12] are at $589 \mathrm{~nm}$, where $A_{\text {meas. }}$ is measured at $532 \mathrm{~nm}$. The value of $\rho(\mathrm{dn} / \mathrm{d} \rho)$ given in [7] is at $546 \mathrm{~nm}$. Notice that the simple estimate for $\mathrm{A}_{\text {meas }}$. gives reasonable agreement with experimentally determined values above the HEL.

\begin{tabular}{llcccc}
\hline \multicolumn{1}{c}{ Material } & \multicolumn{1}{c}{$\mathrm{n}_{0}$} & $\rho(\mathrm{dn} / \mathrm{d} \rho)$ & $\mathrm{A}_{\text {calc. }}=\mathrm{n}_{0}-\rho(\mathrm{dn} / \mathrm{d} \rho)$ & $\mathrm{A}_{\text {meas. }}=\mathrm{u}_{\mathrm{a}} / \mathrm{u}_{0}$ & Above / Below HEL? \\
\hline $\mathrm{Al}_{2} \mathrm{O}_{3}$ & $1.768\left(\mathrm{n}_{\text {ord. }}\right)$ & $-0.245^{[12]}$ & 2.013 & $1.787^{[10]}$ & below \\
$\mathrm{LiF}$ & 1.393 & $0.13^{[8]}$ & 1.26 & $1.286^{[6]}$ & above \\
$\mathrm{MgO}$ & 1.742 & $-0.267^{[7]}$ & 2.007 & 1.560 & below \\
$\mathrm{MgO}$ & 1.742 & $-0.267^{[7]}$ & 2.007 & 1.978 & above \\
Quartz & $1.544\left(\mathrm{n}_{\text {ord. }}\right)$ & $-0.392^{[11]}$ & 1.936 & $1.081^{[4]}$ & below \\
\hline
\end{tabular}




\section{ACKNOWLEDGEMENTS}

We would like to thank the following colleagues who helped execute and interpret the experiments: Bill Anderson, John Vorthman, Dennis Hayes, Dennis Shampine, Jim Esparza, and Mark Byers.

\section{REFERENCES}

1. Barker, L. M., and Hollenback, R. E., "Laser Interferometer for Measuring High Velocities of any Reflective Surface", J. of Applied Physics 43, 4669, (1972).

2. Hemsing, W. F., "Velocity Sensing Interferometer (VISAR) modification”, Rev. Sci. Instrum 50, 73 (1979)

3. Barker, L. M. and Hollenbach, R. E., "Shock Wave Studies of PMMA, Fused Silica, and Sapphire, J. Appl. Phys 41, 4208 (1970).

4. Jones, S. C. and Gupta, Y. M., "Refractive Index and Elastic Properties of Z-Cut Quartz shocked to 60 kbar”, J. Appl. Phys. 88, 5671 (2000)

5. Duffy, T. S. and Ahrens, T. J. "Compressional Sound Velocity, Equation of State, and Constitutive Response of Shock-compressed Magnesium Oxide", J. Geophys. Res. 100, 529 (1995).

6. Hayes, D., "Unsteady Compression Waves in Interferometer Windows", J. Appl. Phys. 89, 6484, (2001).

7. Vedam, K. and Schmidt, E. D. D., "Variation of Refractive Index of $\mathrm{MgO}$ with Pressure to 7 kbar", Phys. Rev. 146, 548 (1966).
8. Waxler, R. M. and Weir, C. E., "Effects of hydrostatic pressure upon the refractive index of some solids", J. Res. Nat. Bur. Stand., 69A, 325 (1965).

9. S.P. Marsh ed., LASL Shock Hugoniot Data, p. 312, University of California Press, Berkeley, 1980.

10. A linear, least squares fit to the raw data of Setchell, R. E. "Refractive index of sapphire at $532 \mathrm{~nm}$ under shock compression and release", J. Appl. Phys. 91, 2833 (2002).

11. Vedam, K. and Davis, T. A. "Nonlinear variation of the refractive indicies of a Quartz with pressure", J. Opt. Soc. Am. 571140 (1967).

12. Davis, T. A. and Vedam, K. "Photo-elastic properties of sapphire $\left(\alpha-\mathrm{Al}_{2} \mathrm{O}_{3}\right)$ ”, J. Appl. Phys. 38, 4555 (1967).

\section{COPYRIGHT}

This manuscript has been authored by Bechtel Nevada under Contract No. DE-AC08-96NV11718 with the U.S. Department of Energy. The United States Government retains and the publisher, by accepting the article for publication, acknowledges that the United States Government retains a nonexclusive, paid-up, irrevocable, world-wide license to publish or reproduce the published form of this manuscript, or allow others to do so, for United States Government purposes. 